\title{
Effects of Thyroid Hormones on Skeletal Muscle Bioenergetics In Vivo Phosphorus-31 Magnetic Resonance Spectroscopy Study of Humans and Rats
}

\author{
Zohar Argov, Perry F. Renshaw, Barry Boden, A. Winokur, and William J. Bank \\ Departments of Neurology, Biochemistry/Biophysics and Pharmacology, University of Pennsylvania School \\ of Medicine, Philadelphia, Pennsylvania 19104
}

\begin{abstract}
The pathophysiology of the myopathy in dysthyroid states is poorly understood. We therefore tested the effects of thyroid hormones on muscle bioenergetics in humans and rats, using in vivo ${ }^{31} \mathrm{P}$ NMR. Two hypothyroid patients had: low phosphocreatine to inorganic phosphate ratio $(\mathrm{PCr} / \mathrm{Pi})$ at rest, increased $\mathrm{PCr}$ depletion during exercise and delayed postexercise recovery of $\mathbf{P C r} / \mathrm{Pi}$. Eight thyroidectomized rats did not show abnormalities at rest, but muscle work induced by nerve stimulation resulted in a significantly $(P<0.0001)$ lower $P C r /$ $\mathrm{Pi}$ (35-45\% of control) at each of the three stimulation frequencies tested $(0.25,0.5$, and $1.0 \mathrm{~Hz})$. Recovery rate was markedly slowed to one-third of normal values. Thyroxine therapy reversed these abnormalities in both human and rat muscle. Five patients and six rats with hyperthyroidism did not differ from normal controls during rest and exercise but had an unusually rapid recovery after exercise. The bioenergetic abnormalities in hypothyroid muscle suggest the existence of a hormone-dependent, reversible mitochondrial impairment in this disorder. The exercise intolerance and fatigue experienced in hypothyroid muscle may be due to such a bioenergetic impairment. The changes in energy metabolism in hyperthyroid muscle probably do not cause the muscular disease in this disorder.
\end{abstract}

\section{Introduction}

Skeletal muscle is a major target organ of thyroid hormone. Muscle weakness and fatigue are common to both hypothyroidism and hyperthyroidism in man $(1,2)$. Although many structural and biochemical abnormalities have been identified in muscle during the dysthyroid state, in both humans and animals, it is not clear which of these result in the impairment of muscle function $(1,2)$.

In hypothyroidism transformations in the biochemical state of muscle occur, as myosin develops slow fiber characteristics (3). Furthermore, the activities of several key mitochondrial enzymes are reduced (4-9). The latter is more pronounced in slow twitch, red muscles compared with fast, white muscles $(7,8)$. Oxidative metabolism of these transformed

Presented in part before the 39th Meeting of the American Academy of Neurology, New York, April 1987.

Address reprint requests to Dr. William Bank, Department of Neurology, Hospital of the University of Pennsylvania, 3400 Spruce Street, Philadelphia, PA 19104.

Received for publication 9 April 1987 and in revised form 26 October 1987.

J. Clin. Invest.

(c) The American Society for Clinical Investigation, Inc.

$0021-9738 / 88 / 06 / 1695 / 07 \$ 2.00$

Volume 81, June 1988, 1695-1701 fibers should be less efficient in hypothyroidism, yet Wiles et al. $(10,11)$ found diminished fatigability in human hypothyroid muscles. The reduction of isometric force in hypothyroid muscle stimulated at $20 \mathrm{~Hz}$ for 2 min was $<20 \%$ of its initial values, compared with $60 \%$ reduction in controls (11). Furthermore, these investigators suggested that energy metabolism is more efficient in hypothyroidism. ATP turnover was found to be reduced in working human hypothyroid muscles $(10,11)$ and similar data were obtained from tetanized animal muscles $(12,13)$. A reduction in mitochondrial activity and improved bioenergetics (by a factor of 3 ) is paradoxical and inconsistent with the fact that many hypothyroid patients complain of exercise intolerance and fatigue $(1,2)$.

The above experiments, showing reduced fatigability and increased efficiency of hypothyroid muscles, were performed under ischemic conditions (in animals under prolonged tetanic stimulation, where muscle is also working ischemically) and do not adequately reflect the aerobic conditions in working muscle. Such conditions were not studied in hypothyroidism and we therefore examined aerobically working hypothyroid muscle in both humans and rats in vivo.

Increased thyroid hormone levels result in increased mitochondrial enzymes activity in muscle and this is again more pronounced in slow fibers $(8,14-17)$. Muscle bioenergetic studies have been less conclusive in hyperthyroidism; ATP turnover was increased in a single case compared with controls (10), while rats showed no significant changes (12). Although hyperthyroidism may also present with exercise intolerance, it is not clear whether the biochemical findings relate to the clinical presentation. The bioenergetic disorder in both hypo- and hyperthyroidism is not well understood and very likely is due to different mechanisms.

In order to address this issue we used phosphorus nuclear magnetic resonance spectroscopy $\left({ }^{31} \mathrm{P} \mathrm{NMR}\right)^{1}$ to study skeletal muscle in vivo in both hypo- and hyperthyroidism. This technique provides a noninvasive method for studying the bioenergetic state of muscle during transitions from rest to work and is most useful in evaluating oxidative metabolism in working muscle under normal aerobic conditions (18-21).

We have addressed the following questions: Is there a bioenergetic impairment in muscles during both dysthyroid states in vivo? What is the nature of this impairment? Are changes in energy metabolism reversible with therapy and can ${ }^{31} \mathrm{P}$ NMR be used to monitor muscle disease in thyroid abnormalities?

\section{Methods}

\section{Patients}

Hypothyroidism. Two patients with hypothyroid myopathy were studied. Patient $A$ was a 37-yr-old construction worker with primary hypo-

1. Abbreviations used in this paper: FID, free induction decay; NMR, nuclear magnetic resonance. 
thyroidism, who complained of excessive fatigue, cramps, and "hardening of muscles." He had mild muscle weakness; his deltoid, biceps, and iliopsoas muscles could be overcome by the examiner (grade 4+ MRC scale). Loss of hair, dryness of hands, puffiness of the face were also found and he gained $15 \mathrm{~kg}$ over a few months. Serum CK was $3239 \mathrm{IU} /$ liter (normal $<140$ ), $\mathrm{T}_{4}=0.4 \mu \mathrm{g} / \mathrm{dl}$ (normal range 4.2-11.5) and TSH $=326 \mathrm{IU} / \mathrm{ml}$ (normal range 0.5-5). Electromyogram (EMG) was normal. Muscle biopsy showed mild myopathic changes (variation in fiber size and central nuclei) in several areas. He was tested by ${ }^{31} \mathbf{P}$ NMR (see below) twice before therapy and then given thyroid replacement (L-thyroxine, $0.15 \mathrm{mg} / \mathrm{d}$ ). Gradual clinical and laboratory improvement was noted over a period of $8 \mathrm{wk}$; CK fell to 89 and TSH to 11.2 while $T_{4}$ rose to 5.2. Biopsy was not repeated. He was tested by ${ }^{31} \mathrm{P}$ NMR on five occasions during this period. After 5 mo he made a full recovery and returned to work.

Patient B was a 30-yr-old woman with hypothyroidism secondary to ${ }^{131} I$ therapy for past hyperthyroidism. She complained of pain, tenderness and "cramping" of her thigh muscles and soreness of her calf muscles after mild exercise. Her muscles were firm on palpation and she had a waddling gait but there was no clear muscle weakness elsewhere. EMG was normal and muscle biopsy showed subsarcolemmal deposits of storage material (probably mucopolysaccharides-2). At the time of the first ${ }^{31} \mathrm{P}$ NMR test serum CK was $245 \mathrm{IU} / \mathrm{liter}, \mathrm{T}_{4}=2.9$ $\mu \mathrm{g} / \mathrm{dl}$ and $\mathrm{TSH}=32.1 \mathrm{IU} / \mathrm{ml}$. Therapy with L-thyroxine $(0.125 \mathrm{mg} / \mathrm{d})$ was begun and $\mathrm{T}_{4}$ and TSH returned to normal levels. She was tested twice during this period.

Hyperthyroidism. Five patients with laboratory evidence of increased $\mathrm{T}_{4}(13-15.5 \mu \mathrm{g} / \mathrm{dl})$ were tested once during their hyperthyroid state. Only one had clinical evidence of myopathy (muscle weakness, muscle atrophy) and all came to the attention of their physicians because of nonmuscular complaints. They were not tested after therapy.

Control subjects. Normal controls for the NMR tests were 22 volunteers (investigators and technicians working in the laboratory) between the ages of 20-50 yr who were sedentary or only engaged in recreational sports. Normal controls were symptom free for any disease, and several subjects had $\mathrm{T}_{4}$ determinations that were normal. All subjects gave informed consent for these studies.

\section{Animals}

Hypothyroid. Eight male rats $(225 \pm 25 \mathrm{~g}$ body wt) had their thyroid glands surgically removed (commercially obtained from Charles River Laboratories, Wilmington, MA). They were maintained in a hypothyroid state for 2 mo before testing. Studies have shown that after 4-6 wk of hypothyroidism due to thyroidectomy, biochemical and physiological changes occur in muscles $(4,9,15)$. Body weight increased only slightly to $245 \pm 10 \mathrm{~g}$ during this 2-mo period (normal rats gained $100-150 \mathrm{~g}$ during the same time), a feature consistant with other studies of rat hypothyroidism (8). Serum $\mathrm{T}_{4}$ levels were undetectable at the time of NMR testing (normal 6.05 $\pm 1.55 \mu \mathrm{g} / \mathrm{dl}, \pm 2 \mathrm{SD}, n=16$ ). After the animals were evaluated by NMR, they received supplemental Lthyroxine in the drinking water $(0.5 \mathrm{mg} / \mathrm{liter})$. Body weight then increased to 290-315 g after $6 \mathrm{wk}$ of therapy, at which point they were retested by ${ }^{31} \mathrm{P}$ NMR. Blood samples taken at the end of each experiment showed $\mathrm{T}_{4}$ values slightly higher than normal $(8.0 \pm 1.0 \mu \mathrm{g} / \mathrm{dl})$.

Hyperthyroid. Six normal rats were given a high L-thyroxine load in the drinking water ( $4 \mathrm{mg} /$ liter) for $6 \mathrm{wk}$ before testing (22). Serum $T_{4}$, determined from blood samples taken at the time of the ${ }^{31} \mathrm{P}$ NMR experiment, was markedly elevated in each of the animals to an average of $19 \pm 4.5 \mu \mathrm{g} / \mathrm{dl}$. Body weight increased as in normal animals by $100 \pm 20 \mathrm{~g}$. Chronic oral thyroxine loading in rats results in significantly increased basal metabolic rate as well as changes in muscle enzymes $(17,22,23)$.

Control. 20 normal rats with weights ranging from 250 to $350 \mathrm{~g}$ and similar ages to the diseased animals were tested by ${ }^{31} \mathrm{P}$ NMR, using protocols similar to those used in the diseased animals (24). All animals (normal and diseased) had free access to a standard pellet diet and water. Two rats were kept in one cage, and all animals seemed alert and active to similar degrees when examined biweekly. Handling of all animals was in accordance with the University of Pennsylvania guidelines for animal care.

\section{Serum $T_{4}$ determination}

Samples of blood $\left(0.5 \mathrm{~cm}^{3}\right)$ were taken from the tail vein of anesthetized rats immediately after the NMR experiment. $T_{4}$ was determined by radioimmunoassay, using a commercially available kit (Magic $T_{4}$ RIA No. 472300; Corning Medical Products, Inc., Corning, NY).

\section{${ }^{31} P N M R$}

The basic protocols and techniques used in our laboratory for testing human arm muscle and rat hindleg muscles by NMR have been described in previous publications (see below). These procedures will, therefore, only be described briefly.

Human arm muscle. A double-tuned, 4-cm diam, two-turn surface coil was used $(18,25,26)$. The coil was placed under the belly of the flexor muscle group of the forearm, which performs the work during wrist flexion exercise against a Cybex II ergometer. Patients were examined in a 1.9 tesla, $30-\mathrm{cm}$ bore magnet coupled to a spectrometer (TMR-32; Oxford Research System, Oxford, England) with an operating frequency of $32.5 \mathrm{MHz}$ for phosphorus. Spectra were obtained by free induction decay (FID) using $90^{\circ}$ pulse angle (40 $\mu$ s duration) with pulse repetition time of $5 \mathrm{~s}$. Spectra were collected at rest (60 sequentional scans averaged during a 5-min period), during exercise against the ergometer (12 scans per min averaged in 1-min blocks) and during postexercise recovery (in 0.5 -min blocks of six scans).

Initially, the patient's maximal torque against the ergometer was measured. Subjects then exercised for 3 min at maximal torque, after which postexercise recovery was monitored. After $30 \mathrm{~min}$ of rest, exercise was resumed at $50 \%$ of the maximal torque and maintained for 7-8 min (the time needed to achieve a short-term steady state). Postrecovery was again monitored. In both above exercise protocols, $0.5 \mathrm{~s}$ duration contractions performed every $5 \mathrm{~s}$ were used and these were synchronized with the NMR sampling through verbal commands. The command for initiation of the 0.5 -s contraction was given immediately after the FID was collected. NMR data was therefore collected after the muscle had returned to its precontraction position. The $50 \%$ of maximal capacity protocol yields comparable results, expressed as end exercise $\mathrm{PCr} / \mathrm{Pi}$, in normal subjects who differ in their maximal torque (muscle power), but can maintain exercise at the $50 \%$ level for 7-10 min.

Animal hindleg protocol. A solenoid coil was placed around the left hindleg of anesthetized rats (pentobarbital, $30-40 \mathrm{mg} / \mathrm{kg}$ weight i.p.) (24). This coil recorded spectra only from the muscles below the knee (anterior compartment and calf). Radio frequency pulse characteristics were: $18 \mu$ s duration $\left(90^{\circ}\right)$ given every $3 \mathrm{~s}$. Spectra were collected in 2-min blocks (40 sequentional scans) during rest and exercise and in 1-min blocks during recovery. Muscle work (exercise) was induced by sciatic nerve stimulation at rates of $0.25,0.5$, and $1.0 \mathrm{~Hz}$ for the hypothyroid rats and $0.25,0.5,1.0$, and $2.0 \mathrm{~Hz}$ for normal and hyperthyroid rats. Postexercise recovery was monitored immediately after the end of stimulation. To assure similar levels of $\mathrm{PCr}$ depletion in all animals (end exercise $\mathrm{PCr} / \mathrm{Pi}$ range of $0.6-0.9$ ), the last frequency in the hypothyroid rats was lower than in normal controls.

Data analysis. Spectra were apodized with $10 \mathrm{~Hz}$ of line broadening, Fourier transformed, phased and plotted with an $X-Y$ plotter. A common baseline for all peaks was drawn by connecting the midnoise level between gamma and beta ATP peaks and peak areas were measured. Data analysis of all ${ }^{31} \mathrm{P}$ NMR spectral peaks' area was done by triangulation to this baseline (Fig. 1). The beta ATP was used for all calculations concerning ATP levels. Intracellular pH was measured from the ${ }^{31} \mathrm{P}$ NMR spectrum (27). The postexercise recovery rate was calculated for the period of $0.5-2.5 \mathrm{~min}$ after recovery, because the slope remains linear only during this time period (25). Nonpaired Student $t$ tests were used for statistical comparisons. 


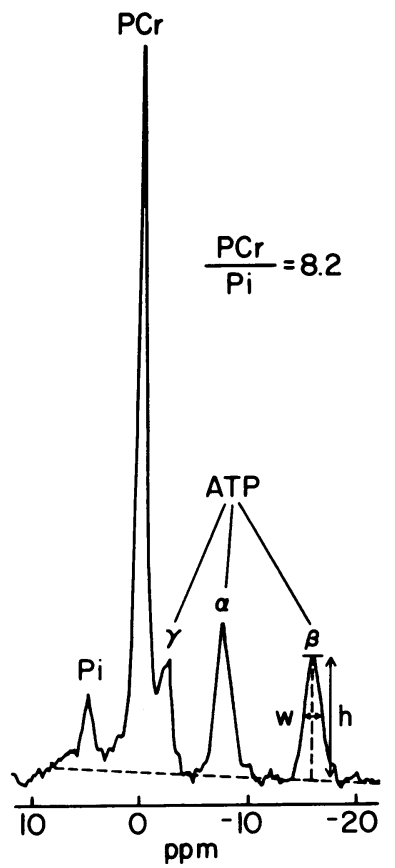

Figure 1. Normal human ${ }^{31} \mathrm{P}$ NMR spectrum of muscle at rest (60 scans at $5 \mathrm{~min}$ ). The peaks of phosphosphocreatine ( $\mathrm{PCr}$ ), inorganic phosphate $(\mathrm{Pi})$, and three phosphates of ATP are identified. A baseline was drawn (broken line) from which all peak heights were measured. The width at half height $(\mathrm{W}) \mathrm{X}$ peak height $(\mathrm{H})$ was used for area calculation (see markings on beta ATP peak). PCr is not completely separated from gamma ATP in human studies at 1.9 tesla. However, our calculations for its area related it to the common baseline; the peak height was also measured from this line.

\section{Results}

\section{Human muscle}

Muscle at rest. ${ }^{31} \mathrm{P}$ NMR spectra of normal muscle at rest (Fig. 1) show a high ratio of phosphocreatine to inorganic phosphate $(\mathrm{PCr} / \mathrm{Pi})$. In 20 control subjects this ratio averaged $8.5 \pm 2.1$, the Pi being about two-thirds of the ATP (range 0.4-0.85). In both patients with hypothyroid myopathy, muscle at rest had a low PCr/Pi of 4.8 and 5.5 (Fig. $2 a$ and Table I). These low values were probably due to an increase in inorganic phosphate (Pi/ATP was $>1$ in both patients), as the
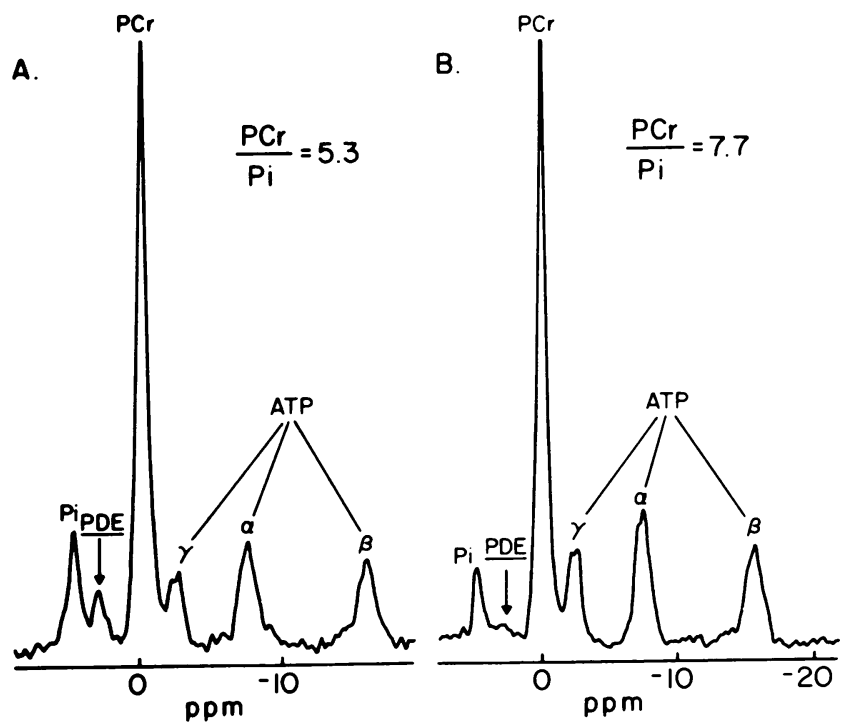

Figure $2 .{ }^{31} \mathrm{P}$ NMR spectra of hypothroid human muscle at rest. Data from patient A before $(A)$ and after $10 \mathrm{wk}$ of hormonal replacement $(B)$. Note the increase in $\mathrm{PCr} / \mathrm{Pi}$ and disappearance of phosphodiester (PDE) peak with therapy.
Table I. Summary of the ${ }^{31} P$ NMR Results in Two Hypothyroid Myopathy and Five Hyperthyroid Patients

\begin{tabular}{lccc}
\hline & \multicolumn{2}{c}{$\mathrm{PCr} / \mathrm{Pi}$} & \\
\cline { 2 - 3 } & Rest & $50 \%$ Max exercise & Recovery rate* \\
\hline $\begin{array}{l}\text { Patient 1 } \\
\quad\end{array}$ & & \\
$\quad$ Before T4 & $4.8-5.3^{*}$ & $0.65^{*}$ & $1.1^{*}$ \\
$\quad$ After T4 & 8.1 & 1.25 & 2.5 \\
Patient 2 & & & \\
$\quad$ Before T4 & $5.5^{*}$ & $\mathrm{ND}$ & $0.5^{*}$ \\
$\quad$ After T4 & 9.0 & 1.02 & 1.5 \\
Hyperthyroidism \pm 2 SD & $7.8 \pm 1.0$ & $1.25 \pm 0.2$ & $5.0 \pm 0.8^{* *}$ \\
Control \pm 2 SD & $8.5+2.1$ & $1.1+0.3$ & $1.8+0.35$ \\
& & & \\
\hline
\end{tabular}

${ }^{*} P<0.02$ compared with control values.

** $P<0.01$ compared with control values of $3.0 \pm 0.7$ for similar depleting exercise.

${ }^{*}$ In $\mathrm{PCr} / \mathrm{Pi} \mathrm{U}$ per min.

ND, Not done.

PCr/ATP values remained in the normal range; 5.0 and 5.2 in patients $A$ and $B$, respectively (average controls: $5.5 \pm 0.7 ; \pm 2$ $\mathrm{SD}, n=20$ ). An abnormal phosphodiester peak was found in one hypothyroid patient (Fig. $2 a$ ). In the five hyperthyroid patients no abnormality in the ${ }^{31} \mathrm{P}$ NMR muscle spectrum was observed at rest.

Therapy led to an improvement of the $\mathrm{PCr} / \mathrm{Pi}$ at rest in both hypothyroid myopathy patients (Fig. $2 b$ and Table I). The $\mathrm{PCr} / \mathrm{Pi}$ returned to normal range after 1 mo of hormonal replacement (Fig. 3). The abnormal phosphodiester peak, which was observed during the hypothyroid state in patient $A$, also disappeared with therapy (Fig. $2 b$ ).

Exercising muscle. During exercise $\mathrm{PCr}$ falls and $\mathrm{Pi}$ rises in any working muscle, but the sum of $\mathrm{PCr}+\mathrm{Pi}$ remained constant. In a group of 22 controls, exercise at $50 \%$ of maximal capacity resulted in an average $\mathrm{PCr} / \mathrm{Pi}$ of $1.1 \pm 0.3( \pm 2 \mathrm{SD})$. In patient $\mathrm{A}$, the $\mathrm{PCr} / \mathrm{Pi}$ fell to 0.65 at the end of similar exercise, while patient $B$ could not maintain a steady state even at $50 \%$ of maximal capacity. In both control group and patient A no drop in ATP was observed. Therapy improved the exercise bioenergetics in both patients, when their serum $T_{4}$ levels returned to normal (Table I). Patient A also exercised against the

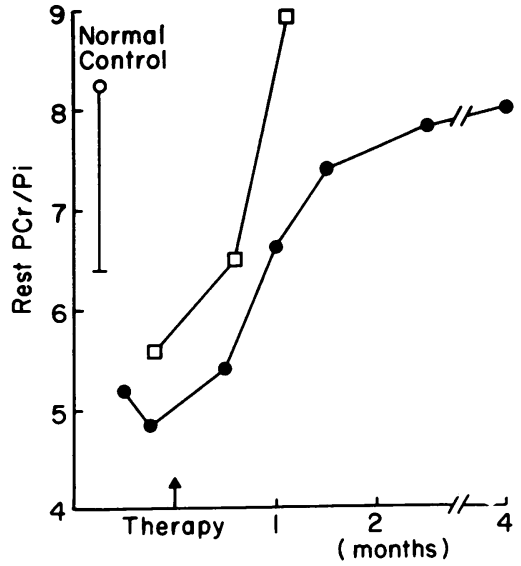

Figure 3. Time course of therapy-induced improvement in $\mathrm{PCr} / \mathrm{Pi}$ at rest in two patients with hypothyroid myopathy. 
ergometer at two work loads once before and once after $10 \mathrm{wk}$ of hormonal replacement. During this period his maximal torque (single contraction power) remained unchanged. The slope of work rate to $\mathrm{Pi} / \mathrm{PCr}$ improved from $14 \mathrm{~J} / \mathrm{min}$ per $\mathrm{Pi} / \mathrm{PCr}$ to $48 \mathrm{~J} / \mathrm{min}$ per $\mathrm{Pi} / \mathrm{PCr}$ (Fig. 4). This exercise slope permits better comparison of different exercise performances (19-21) and has a normal range of 30 to $50 \mathrm{~J} / \mathrm{min}$ per $\mathrm{Pi} / \mathrm{PCr}$.

Hyperthyroid patients $(n=4)$ did not exhibit any ${ }^{31} \mathrm{P}$ NMR abnormality with exercise at $50 \%$ of maximal capacity (endexercise $\mathrm{PCr} / \mathrm{Pi}$ was $1.25 \pm 0.2$ ). The change in $\mathrm{pH}$ during maximal exercise was normal in both hypothyroid and hyperthyroid muscle (pH at end of exercise 6.6-6.8). No accumulation of sugar phosphates was observed.

Postexercise recovery. The rate of postexercise $\mathrm{PCr} / \mathrm{Pi}$ recovery is dependent upon the degree of $\mathrm{PCr}$ depletion at the end of exercise (28). When end-exercise $\mathrm{PCr} / \mathrm{Pi}$ is $0.5-0.9$, the recovery rate is normally $1.8 \pm 0.35( \pm 2 \mathrm{SD}) \mathrm{PCr} / \mathrm{Pi} \mathrm{U} / \mathrm{min}$. The rate increases to $3.0 \pm 0.7( \pm 2 \mathrm{SD}) \mathrm{U} / \mathrm{min}$ when the endexercise $\mathrm{PCr} / \mathrm{Pi}$ is $1.0-1.2$. The recovery rate was significantly slow in patient $\mathrm{A}$ before therapy (end-exercise $\mathrm{PCr} / \mathrm{Pi}=0.5$ ) and gradually returned to normal values after $6 \mathrm{wk}$ of thyroxine supplement (end-exercise was $\mathrm{PCr} / \mathrm{Pi}=0.8$ ) (Table I). The postexercise recovery rate of patient $\mathrm{B}$ was also slow before therapy, and normalized after 1 mo of hormonal replacement (end-exercise $\mathrm{PCr} / \mathrm{Pi}$ was similar on both tests at 0.7 ) (Table I). The recovery rates of the $\mathrm{PCr} / \mathrm{Pi}$ were abnormally fast in all the five hyperthyroid patients, ranging between 4.2 and $5.8 \mathrm{U} / \mathrm{min}$ (end-exercise $\mathrm{PCr} / \mathrm{Pi} 1.1 \pm 0.1, \pm 2 \mathrm{SD}$ ).

\section{Animal muscle}

Muscle at rest. ${ }^{31} \mathrm{P}$ NMR spectra at rest did not show any significant abnormality in the hypothyroid or hyperthyroid rats. The ratio of $\mathrm{PCr} / \mathrm{Pi}$ was $7.6 \pm 2.4( \pm 2 \mathrm{SD}, n=8)$ in the hypothyroid rat muscle and $8.0 \pm 1.5( \pm 2 \mathrm{SD}, n=6)$ in the hyperthyroid muscle (normal $6.8 \pm 2.0, n=20$ ). No abnormal spectral peaks were observed in rats with either dysthyroid condition.

Working muscle. During muscle work induced by nerve stimulation the $\mathrm{PCr} / \mathrm{Pi}$ ratio decreased to lower levels in the hypothyroid rats compared with normal animals (Fig. 5), while in both groups stoichiometric changes between $\mathrm{PCr}$ and $\mathrm{Pi}$ were maintained (the amount of $\mathrm{PCr}$ drop was equal to the

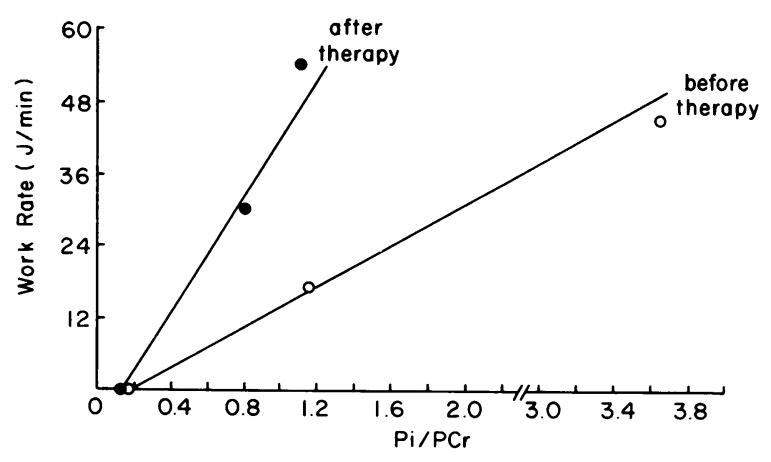

Figure 4. Work rate to $\mathrm{Pi} / \mathrm{PCr}$ slope in patient $\mathrm{A}$ before and $10 \mathrm{wk}$ after hormonal replacement. The patient exercised at two levels in each test, allowing the contraction of this function. The patient's maximal torque has changed by $<8 \%$ during this period, thus the slopes are comparable.

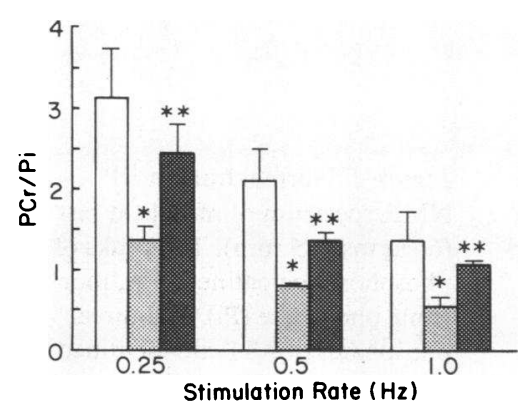

Pi rise, hence their sum remained constant). These differences were significant $(P<0.001)$ at each of the three frequencies used. ATP levels at the end of stimulation protocol were $98 \pm 6 \%$ of their resting values in the hypothyroid muscles (controls $104 \pm 5 \%$, differences were not significant). Six weeks of replacement therapy led to marked improvement of this abnormality; the average ratios rising by $70-91 \%$, although they did not return to normal values (Fig. 5). The PCr/Pi ratios of hyperthyroid muscle were within control range at each of the four levels of work (Fig. 6). pH falls only slightly from 7.15 to 7.02 in normal rats during the stimulation (24). Similar changes were observed in both hypothyroid and hyperthyroid rats. No accumulation of sugar phosphates was noted.

Postexercise recovery. Recovery from exercise was markedly slowed in the hypothyroid animal muscle (Fig. 7); $\mathrm{PCr} / \mathrm{Pi}$ returned to normal levels only after 3-4 min (normal 1.5-2.0 $\mathrm{min}$ ) with an average initial slope of $0.9 \pm 0.2( \pm 2 \mathrm{SD}) \mathrm{PCr} / \mathrm{Pi}$ $\mathrm{U} / \mathrm{min}$ (normal slope from similar end exercise depletion was $2.7 \pm 0.8, P<0.001)$. Therapy more than doubled the recovery rate to $1.9 \pm 0.45 \mathrm{U} / \mathrm{min}(P<0.05$ compared to before therapy), but did not achieve normal values. In hyperthyroid muscle the recovery rate was very rapid with an average slope of $3.9 \pm 0.5 \mathrm{U} / \mathrm{min}(P<0.01$, compared to control $)$ and reached normal resting range in $<1.5 \mathrm{~min}$.

\section{Discussion}

${ }^{31} \mathrm{P}$ NMR is a particularly useful technique for studying tissue bioenergetics in vivo (18-21). This technique has been used in the evaluation of metabolic disorders of muscle that present clinically with exercise intolerance and are associated with defects in energy metabolism $(29,30)$. Since patients with hypo-

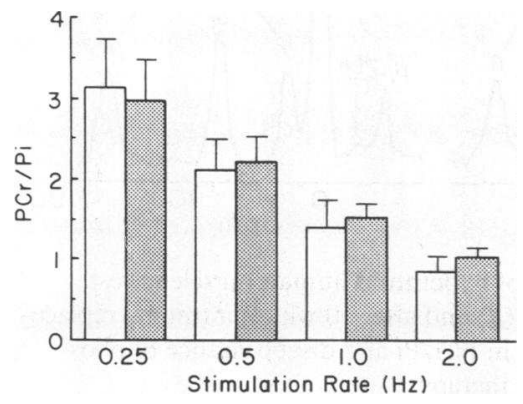

Figure 6. Average $\mathrm{PCr} /$ $P i$ levels at the end of each stimulation period in normal control ( $\square$ ) rats and hyperthyroid rats $(T x, \square)$. The values were not significantly different. Bars $= \pm 2$ SD. 


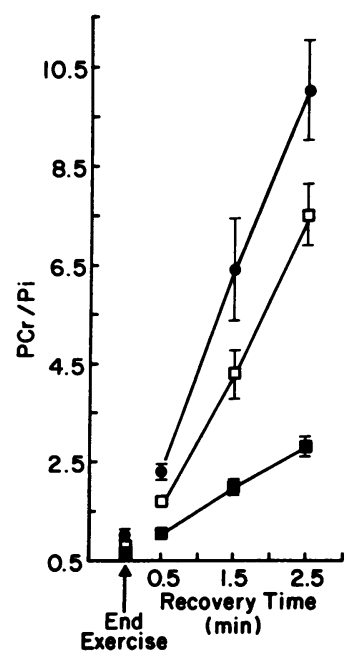

Figure 7. Postexercise recovery of $\mathrm{PCr} / \mathrm{Pi}$ in control (ם), HT ( $\square$ ), and Tx $(\bullet)$ rats. Each point is average for all the group, bars $= \pm$ standard error of the mean. The slope was calculated for the linear portion only $(0.5-2.5$ $\mathrm{min})$. Note that the end-exercise $\mathrm{PCr} /$ $P i$ levels were comparable in the various animals.

thyroidism complain of fatigue, exercise intolerance, and exertional myalgia $(1,2)$, we felt that ${ }^{31} \mathrm{P}$ NMR might detect bioenergetic abnormalities in this disorder.

The ratio of $\mathrm{PCr} / \mathrm{Pi}$ at rest was abnormally low in the two hypothyroid patients and returned to normal within $1 \mathrm{mo}$ in response to therapy. The ${ }^{31} \mathrm{P} N M R$ measured ratio of $\mathrm{PCr} / \mathrm{Pi}$ is considered to be a reflection of the cellular bioenergetic state $(18,31)$ and was first reported to be abnormally low in mitochondrial myopathies $(25,32)$. Low $\mathrm{PCr} / \mathrm{Pi}$ at rest is, however, not disease specific and has been found in several myopathies that are associated with advanced muscle cell destruction (29, 30). In our patients there was no clinical or biopsy evidence for such widespread cellular damage. The basic mechanisms that lead to this abnormality are not clearly understood, and a variety of factors may be implicated. One factor may be changes in the ratio of slow to fast muscle fibers. In animals, slow twitch, red type 1 muscles have significantly lower PCr/Pi values at rest when compared to fast twitch, white type 2 muscles (33). A biochemical transition of fast to slow fibers does occur in hypothyroid muscles (3). The abnormally low $\mathrm{PCr} / \mathrm{Pi}$ at rest may also be due to mitochondrial malfunction (primary or secondary) that occurs in damaged muscle cells $(25,34)$. Our exercise and recovery studies provide some evidence for an in vivo mitochondrial impairment in hypothyroid muscle (see below). In animals with hypothyroidism, there was no change in $\mathrm{PCr} / \mathrm{Pi}$ at rest. Rat muscle may not show, however, the phenomenon of low $\mathrm{PCr} / \mathrm{Pi}$ at rest in response to other destructive disorders either. Resting $\mathrm{PCr} / \mathrm{Pi}$ improves with therapy or regression of disease in several human muscle disorders: mitochondrial myopathies $(35,36)$, polymyositis $(29)$, carnitine deficiency (37), muscle injury (34), and now hypothyroid myopathy.

Our preliminary observations in the two patients with hypothyroid myopathy also show impaired bioenergetics during exercise and postexercise recovery. The ratio of $\mathrm{PCr}$ to $\mathrm{Pi}$ fell to abnormally low levels during work at $50 \%$ of maximal capacity in one patient and recovered at half the normal rate in both (compared with equal degrees of $\mathrm{PCr}$ depletion). The abnormalities during muscle work and recovery also returned to normal in response to therapy, strengthening the observation that the bioenergetic abnormality in human hypothyroid muscle is hormone dependent.
Rats with hypothyroidism were examined by ${ }^{31} \mathrm{P}$ NMR since they share many metabolic abnormalities of muscle with the human disease. Furthermore, the exercise pattern and postexercise recovery could be monitored during a standard protocol to verify the observations in human muscle. Exercising hypothyroid rat muscle demonstrated an impaired bioenergetic profile: the ratio of $\mathrm{PCr} / \mathrm{Pi}$ fell to abnormally low levels (35-45\% of normal values) during work induced by identical nerve stimulation frequencies and recovered at one-third the normal rate from a comparable end-exercise depletion state. This impaired bioenergetic profile in deficient animals was only partially corrected by hormonal replacement.

Earlier biochemical studies on muscle samples taken during rest and after maximal ischemic exercise showed that work-induced depletion of $\mathrm{PCr}$ and rise in Pi were less pronounced in hypothyroid muscle, both in human and animals (10-13). These findings led to the suggestion that there is less ATP turnover in hypothyroid muscle compared with normal muscle during similar work loads, or that energy metabolism is more efficient in hypothyroidism. These earlier human studies were done in limbs in which circulation was occluded $3 \mathrm{~min}$ before exercise. Animal studies used tetanic nerve stimulation during which blood (and oxygen) supply to muscle is virtually blocked (38). These studies were done in conditions where the supply of energy must rely on glycolytic rather than aerobic metabolism. This may have masked the contribution of defective oxidative metabolism to bioenergetic changes in hypothyroidism. Increased work loads were paradoxically not associated with a further decrease in $\mathrm{PCr} / \mathrm{Pi}$ in these in vitro biochemical determinations (13). A marked rise in Pi was associated with minimal PCr depletion (12), which is not stoichiometric and suggests loss of $\mathrm{PCr}$ from the sample. In vivo investigations demonstrate a stoichiometric relationship between $\mathrm{PCr}$ reduction and rise in $\mathrm{Pi}$ as well as a progressive reduction of $\mathrm{PCr} / \mathrm{Pi}$ during incremental exercise. Our observations do not support the earlier findings in either humans or animals.

The causes of impaired cellular bioenergetics in hypothyroidism may be varied. Possible external factors such as dietary deficiencies and lack of training are not supported by our rat studies. Both control and thyroidectomized rats were of similar weight, had similar diets and gained weight (although the diseased animals did so at a slower rate). Furthermore, ${ }^{31} \mathbf{P}$ NMR measured bioenergetic changes occur only after $6 \mathrm{~d}$ of starvation but not after $3 \mathrm{~d}$ in rats (39), suggesting that only extreme changes in food intake would lead to impaired muscle bioenergetics. Activity was similar for all rats and even intense training for prolonged periods fails to improve the rat muscle ${ }^{31} \mathrm{P}$ NMR profile (McCully, K., unpublished data). A simple transition of fiber types (increase in normal type 1, slow-twitch fibers) also cannot account for all our observations. Muscles with large numbers of type 1 oxidative fibers (e.g., dog muscle) have a very rapid postexercise recovery rate (40), whereas we recorded slow recovery in hypothyroidism. We suggest that the observed bioenergetic abnormalities are due to impaired oxidative metabolism, possibly due to a mitochondrial malfunction in hypothyroidism. The ratio $\mathrm{PCr}$ to $\mathrm{Pi}$ in muscle is primarily controlled by mitochondrial function during the transition from rest to work and especially during postexercise recovery. In primary human mitochondrial disorders, abnormal $\mathrm{PCr} / \mathrm{Pi}$ reduction during exercise and slowed postexercise 
recovery were seen by ${ }^{31} \mathrm{P}$ NMR $(25,32)$. Very similar findings are now also found in vivo in human and rat hypothyroidism.

Evidence that thyroid hormone deficiency has an effect on muscle mitochondrial function has been variable. Electronmicroscopy of hypothyroid muscle suggests an increase in the number of mitochondria (41), while the yield of mitochondrial protein has been reported to be both normal (4) or decreased $(6,8)$. Most authors, however, agree that the activities of those muscle enzymes that serve as markers of mitochondria are reduced in hypothyroidism (4-9). This reduction seems more pronounced in red muscle (7-9) and its net effect on mixed muscles is not clear. Respiratory capacity and oxygen consumption are reduced as a result of the above mitochondrial changes $(6,9,42,43)$. These in vitro experimental data support our hypothesis of a functional impairment of mitochondria in hypothyroidism. This mitochondrial impairment could either be due to enzyme deficiencies or to derangements in the delivery of oxygen and substrates to the mitochondria (or a combination of both). ${ }^{31} \mathrm{P}$ NMR data showed no abnormality in intracellular $\mathrm{pH}$ or ATP levels that would suggest an additional impaired glycolytic activity or loss of ATP in hypothyroidism. The evidence for impaired oxidative metabolism was corrected by hormonal replacement. Thus, we propose that exercise intolerance and fatigue in hypothyroidism may be due to a reversible, hormone-dependent mitochondrial impairment and that muscle bioenergetics are not "hyperefficient" in this disorder.

We did not have an opportunity to test patients with marked muscle involvement and hyperthyroidism. The only abnormality seen in both animal and human muscle during the hyperthyroid state was a very rapid postexercise recovery of the $\mathrm{PCr} / \mathrm{Pi}$. This in vivo finding is consistent with in vitro studies that have shown increased mitochondrial activity in hyperthyroid rat muscle $(8,14-17)$. Rats tested after 6 wk of hyperthyroidism showed no abnormality of $\mathrm{PCr} / \mathrm{Pi}$ during exercise. It is unlikely that impaired bioenergetics are the cause of the myopathy seen in hyperthyroidism, since a more rapid postexercise recovery should not interfere with cellular energy metabolism. The rate of postexercise recovery does however, appear to be a sensitive indicator of mitochondrial function not only in reduced state (25) but also during increased mitochondrial activity.

\section{Acknowledgments}

We thank Jennifer Phillips for her excellent technical assistance.

Supported by grants of the Muscular Dystrophy Association, National Institutes of Health (RR-02305, GM-07170), and the James S. McDonnell Foundation.

\section{References}

1. Ruff, R. L. 1986. Endocrine myopathies (hyper- and hypofunction of adrenal, thyroid, pituitary, and parathyroid glands and iatrogenic steroid myopathy). In Myology: Basic and Clinical. A. G. Engel and B. Q. Banker, editors. McGraw-Hill Book Co., New York. 1871-1906.

2. Layzer, R. B. 1985. Neuromuscular Manifestation of Systemic Disease. F. A. Davis, Philadelphia. 79-98.

3. Ianuzzo, D., P. Patel, V. Chen, P. O'Brien, and C. Williams. 1977. Thyroidal trophic influence on skeletal muscle myosin. Nature (Lond.). 270:74-76.
4. Gollnick, P. D., and C. D. Ianuzzo. 1972. Hormonal deficiencies and the metabolic adaptations of rats to training. Am. J. Physiol. 223:278-282.

5. Baldwin, K. M., A. M. Hooker, R. E. Herrick, and L. F. Schrader. 1980. Respiratory capacity and glycogen depletion in thyroid-deficient muscle. J. Appl. Physiol. 49:102-106.

6. van Hardeveld, C., and A. A. H. Kassenaar. 1978. Effects of experimental hypothyroidism on skeletal muscle metabolism in the rat. Acta Endocrinol. 87:114-124.

7. Nicol, C. J. M., and I. A. Johnson. 1981. Energy metabolism of fast- and slow-twitch skeletal muscle in the rat: thyroid hormone induced changes. J. Comp. Physiol. 142:465-472.

8. Janssen, J. W., C. van Hardeveld, and A. A. K. Kassenaar. 1978. Evidence for a different response of red and white skeletal muscle of the rat in different thyroid states. Acta Endocrinol. 87:768-775.

9. Terjung, R. L., and J. E. Koerner. 1976. Biochemical adaptation in skeletal muscle of trained thyroidectomized rats. Am. J. Physiol. 230:1194-1197.

10. Wiles, C. M., A. Young, D. A. Jones, and R. H. T. Edwards. 1979. Muscle relaxation rate, fibre-type composition and energy turnover in hyper- and hypo-thyroid patients. Clin. Sci. 57:375-384.

11. Wiles, C. M., D. A. Jones, and R. H. T. Edwards. 1981. Fatigue in metabolic myopathy. In Human Muscle Fatigue: Physiological Mechanisms. Pitman Medical, London. 264-282.

12. Leijendekker, W. J., C. van Hardeveld, and A. A. K. Kassenaar. 1983. The influence of the thyroid state on energy turnover during tetanic stimulation in the fast-twitch (mixed type) muscle of rats. Metab. Clin. Exp. 32:615-621.

13. Leijendekker, W. J., C. van Hardeveld, and A. A. K. Kassenaar. 1985. Coupled diminished energy turnover and phosphorylase a formation in contracting hypothyroid rat muscle: Metab. Clin. Exp. 34:437-441.

14. Winder, W. W., and J. O. Holloszy. 1977. Response of mitochondria of different types of skeletal muscle to thyrotoxicosis. Am. J. Physiol. 232:C180-C184.

15. Ianuzzo, C. D., V. Chen, P. O'Brien, and T. G. Keens. 1984. Effect of experimental dysthyroidism on the enzymatic character of the diaphragm. J. Appl. Physiol. 56:117.

16. Kubista, V., J. Kubistova, and D. Pette. 1971. Thyroid hormone induced changes in the enzyme activity pattern of energy-supplying metabolism of fast (white), slow (red), and heart muscle of the rat. Eur. J. Biochem. 18:553-560.

17. Winder, W. W., K. M. Baldwin, R. L. Terjung, and J. O. Holloszy. 1975. Effects of thyroid hormone administration on skeletal muscle mitochondria. Am. J. Physiol. 228:1341-1345.

18. Chance, B., S. Eleff, J. S. Leigh Jr, D. Sokolov, and A. Sapega. 1981. Mitochondrial regulation of phosphocreatine/inorganic phosphate ratios in exercising human muscle: A gated 31-P NMR study. Proc. Natl. Acad. Sci. USA. 78:6714-6718.

19. Chance, B., J. S. Leigh, K. McCully, Z. Argov, and B. Boden. 1986. Evaluation of human exercise performance capability in human limbs. In Problems in the Biochemistry of Physical Exercise and Training. G. Benzi and L. Packer, editors. Elsevier Scientific Publishers, Amsterdam. 491-502.

20. Chance, B., J. S. Leigh Jr, B. J. Clark, J. Maris, J. Kent, S. Nioka, and D. Smith. 1985. Control of oxidative metabolism and oxygen delivery in human skeletal muscle: A steady state analysis of the work/energy cost transfer function. Proc. Natl. Acad. Sci. USA. 82:8384-8388.

21. Chance, B., B. J. Clark, S. Nioka, H. Subramanian, J. Maris, Z. Argov, and $\mathrm{H}$. Bode. 1985. Phosphorus nuclear magnetic resonance spectroscopy in vivo. Circulation. 72(Suppl. 4):103-110.

22. Fundaro, A., L. Molinengo, and M. C. Cassone. 1985. The transition from a fixed ratio to a fixed interval schedule of reinforcement in hypo and hyperthyroid rats. Pharmacol. Res. Commun. 17:463-470. 
23. Fitts, R. H., W. W. Winder, M. H. Brooke, K. K. Kaiser, and J. O. Holloszy. 1980. Contractile, biochemical, and histochemical properties of thyrotoxic rat soleus muscle. Am. J. Physiol. 238:C15-20.

24. Argov, Z., J. Maris, L. Damico, M. Koruda, Z. Roth, J. S. Leigh and B. Chance. 1987. Continuous, graded steady state muscle work in rats studied by in vivo 31-P NMR. J. Appl. Physiol. 63:1428-1433.

25. Argov, Z., W. J. Bank, J. Maris, P. Peterson, and B. Chance. 1987. Bioenergetic heterogeneity of human mitochondrial myopathies as demonstrated by in vivo phosphorus magnetic resonance spectroscopy. Neurology. 37:257-262.

26. Argov, Z., W. J. Bank, J. Maris, J. S. Leigh, and B. Chance. 1987. Muscle energy metabolism in phosphofructokinase deficiency as recorded by 31-P NMR. Ann. Neurol. 22:46-51.

27. Taylor, D. J., P. J. Bore, P. Styles, D. G. Gadian, and G. K. Radda. 1983. Bioenergetics of intact human muscle. A 31-P NMR study. Mol. Biol. Med. 1:77-94.

28. Arnold, D. L., P. M. Mathews, and G. K. Radda. 1984. Metabolic recovery after exercise and the assessment of mitochondrial function in vivo in human skeletal muscle by means of 31 P-NMR. Mag. Reson. Med. 1:301-315.

29. Bank, W., Z. Argov, J. S. Leigh, and B. Chance. 1988. The value of 31-P NMR in the diagnosis and monitoring the course of human myopathies. Ann. NY Acad. Sci. 508:448-450.

30. Edwards, R. H. T., D. R. Wilke, J. M. Dawson, R. E. Gordon, and $D$. Shaw. 1982. Clinical use of nuclear magnetic resonance in the investigation of myopathy. Lancet. i:725-730.

31. Chance, B. 1984. Application of 31-P NMR to clinical biochemistry. Ann. NY Acad. Sci. 428:318-332.

32. Arnold, D. L., D. J. Taylor, and G. K. Radda. 1985. Investigation of human mitochondrial myopathies by phosphorus magnetic resonance spectroscopy. Ann. Neurol. 18:189-196.

33. Meyer, R. A., T. R. Brown, and M. J. Kushmerick. 1985. Phosphorus nuclear magnetic resonance of fast and slow twitch muscle. Am. J. Physiol. 248:C279-C287.

34. McCully, K. K., Z. Argov, B. Boden, R. Brown, W. Bank, and
B. Chance. 1988. Detection of muscle injury in humans with 31-P magnetic resonance spectroscopy. Muscle \& Nerve. 11:212-216.

35. Eleff, S., N. G. Kennaway, N. R. M. Buist, V. M. Darle-Usmar, R. A. Capaldy, W. J. Bank, and B. Chance. 1984. 31 P NMR study of improvement in oxidative phosphorylation by vitamins $\mathrm{K} 3$ and $\mathrm{C}$ in a patient with a defect in electron transport at complex 3 in skeletal muscle. Proc. Natl. Acad. Sci. USA. 81:3529-3533.

36. Argov, Z., W. J. Bank, J. Maris, S. Eleff, N. G. Kennaway, B. Chance, and R. Olson. 1986. Treatment of mitochondrial myopathy due to complex 3 deficiency with vitamins $\mathrm{K} 3$ and C: 31-P NMR follow up study. Ann. Neurol. 19:598-602.

37. Argov, Z., J. Maris, K. Fiscbeck, W. Bank, and B. Chance. 1985. In vivo study of human lipid myopathies by 31-P NMR spectroscopy. Ann. Neurol. 18:119.

38. Gray, S. D., and N. C. Staub. 1967. Resistance to blood flow in leg muscles of dog during tetanic isometric contraction. Am. J. Physiol. 213:677-682.

39. Koruda, M., Z. Argov, J. Maris, R. H. Rolandeli, R. G. Settle, D. O. Jacobs, B. Chance, and J. L. Rombeau. 1985. 31-Phosphorus nuclear magnetic resonance spectroscopy (31-P NMR) of stimulated muscle during starvation. Surg. Forum. 36:61-63.

40. Giger, U., Z. Argov, M. Schnall, and B. Chance. 1986. Myopathy in phosphofructokinase deficient dogs studied by in vivo 31-P NMR. Muscle \& Nerve. 19(Suppl. 5s):187. (Abstr.)

41. Gustafsson, R., J. R. Tata, O. Lindberg, and L. Ernster. 1965. The relationship between the structure and activity of rat skeletal muscle mitochondria after thyroidectomy and thyroid hormone treatment. J. Cell Biol. 26:555-572.

42. Everts, M. E., C. van Hardeveld, H. E. D. J. Ter Keurs, and A. A. K. Kassenaar. 1981. Force development and metabolism in skeletal muscle of euthyroid and hypothyroid rats. Acta Endocrinol. 97:221-225.

43. Baldwin, K. M., S. B. Ernst, R. E. Herrick, A. M. Hooker, and W. J. Mullin. 1980. Exercise capacity and cardiac function in trained and untrained thyroid-deficient rats. J. Appl. Physiol. 49:1022-1026. 